\title{
Meningioma with Intra- and Peritumoral Abscess
}

\author{
Rajesh Reddy Sannareddy ${ }^{1}$ Rahul Lath ${ }^{1} \quad$ Michelle de Padua ${ }^{2} \quad$ Alok Ranjan $^{1}$ \\ ${ }^{1}$ Department of Neurosurgery, Apollo Hospital, Jubilee Hills, \\ Hyderabad, Telangana, India \\ 2Department of Pathology, Apollo Hospital, Jubilee Hills, \\ Hyderabad, Telangana, India \\ Address for correspondence Rajesh Reddy Sannareddy, MCh, \\ Department of Neurosurgery, Apollo Institute of Neurosciences, \\ Jubilee Hills, Hyderabad 500033, Telangana, India \\ (e-mail: rajesh.jipmer@gmail.com).
}

Indian J Neurosurg 2018;7:220-222

\begin{abstract}
The association of meningioma with intra- and peritumoral brain abscess is rare. All cases reported in literature had some focus of infection in the body. We report a 56-year-old man with well-controlled diabetes mellitus found to have a space-occupying

Keywords lesion upon evaluation for headache, vomiting, and clumsiness of right upper limb.

- brain tumor Perioperatively, pus was found intra- and perilesionally around a thick, capsulated, du-

- meningioma ral-based, vascular lesion. Histopathology was suggestive of meningioma, and Esche-

- abscess richia coli was isolated upon culture.
\end{abstract}

\section{Introduction}

Intra- and parasellar tumors are the most common intracranial neoplasms to harbor abscess because of direct extension of microbial flora from paranasal sinuses. ${ }^{1,2}$ Glioblastoma, astrocytoma (high and low grade), ependymoma, and metastatic lesions have also been reported to harbor abscesses within them. Only nine cases of intracranial meningioma associated with brain abscess have been reported in literature so far, with intra- and peritumoral location of abscess being rare. ${ }^{3-5}$ We report yet another case; however, a source of infection could not be identified in our patient.

\section{Case Description}

A 56-year-old man with well-controlled diabetes mellitus was evaluated for headache associated with vomiting and found to have a dural-based, contrast-enhancing lesion with perilesional edema located in relation to middle one-third of the falx on the left side. He refused surgery and presented 7 months later with headache and clumsiness of the right upper limb. Repeat imaging showed increase in the size of the lesion with change in the pattern of contrast enhancement in the area adjacent to the original lesion ( - Fig. 1). Differential diagnoses of meningioma with malignant transformation, metastasis, and tuberculoma were considered.

After obtaining consent, a left temporoparietal craniotomy and excision of the lesion was performed. Perioperatively, the dural-based lesion was vascular with a thick capsule and pus in the center of the lesion that was communicating with the peritumoral abscess ( $\boldsymbol{\sim}$ Fig. 2A). Gross total excision of the lesion with dural attachment was performed. Histopathology was suggestive of meningioma (-Fig. 2B-D). Escherichia coli was isolated from the purulent material. The patient had an uneventful recovery and was treated with antibiotics for 4 weeks.

\section{Discussion}

The vascularity of meningioma, location outside the bloodbrain barrier, and the rich nutritive environment provided by tumor cells make meningiomas susceptible to abscess formation, especially in the presence of foci of infection elsewhere in the body..$^{5-8}$ The vascular pattern of the meningioma is such that the feeding artery supplies the core of the meningioma and the branches supply the periphery facilitating entrapment of pathogen in the center of the tumor. In the presence of additional venous compression by tumor, especially in the parasagittal location, the stagnation of blood and pathogen in the center of the tumor is facilitated. Peritumoral abscess can be explained by the seedling of pathogens during bacteremia at the sites of blood-brain barrier breakdown induced by tumor response. ${ }^{9}$ The patient probably had an untreated, asymptomatic urinary tract infection in the past, which could have precipitated low grade bacteremia, thereby predisposing to intra and peritumoral abscess formation. received

February 26, 2017

accepted after revision

June 8, 2017

published online

October 4, 2017
DOI https://doi.org/

10.1055/s-0037-1606820.

ISSN 2277-954X.
C2018 Neurological Surgeons'

Society of India
License terms

()(1) $\circledast$ 


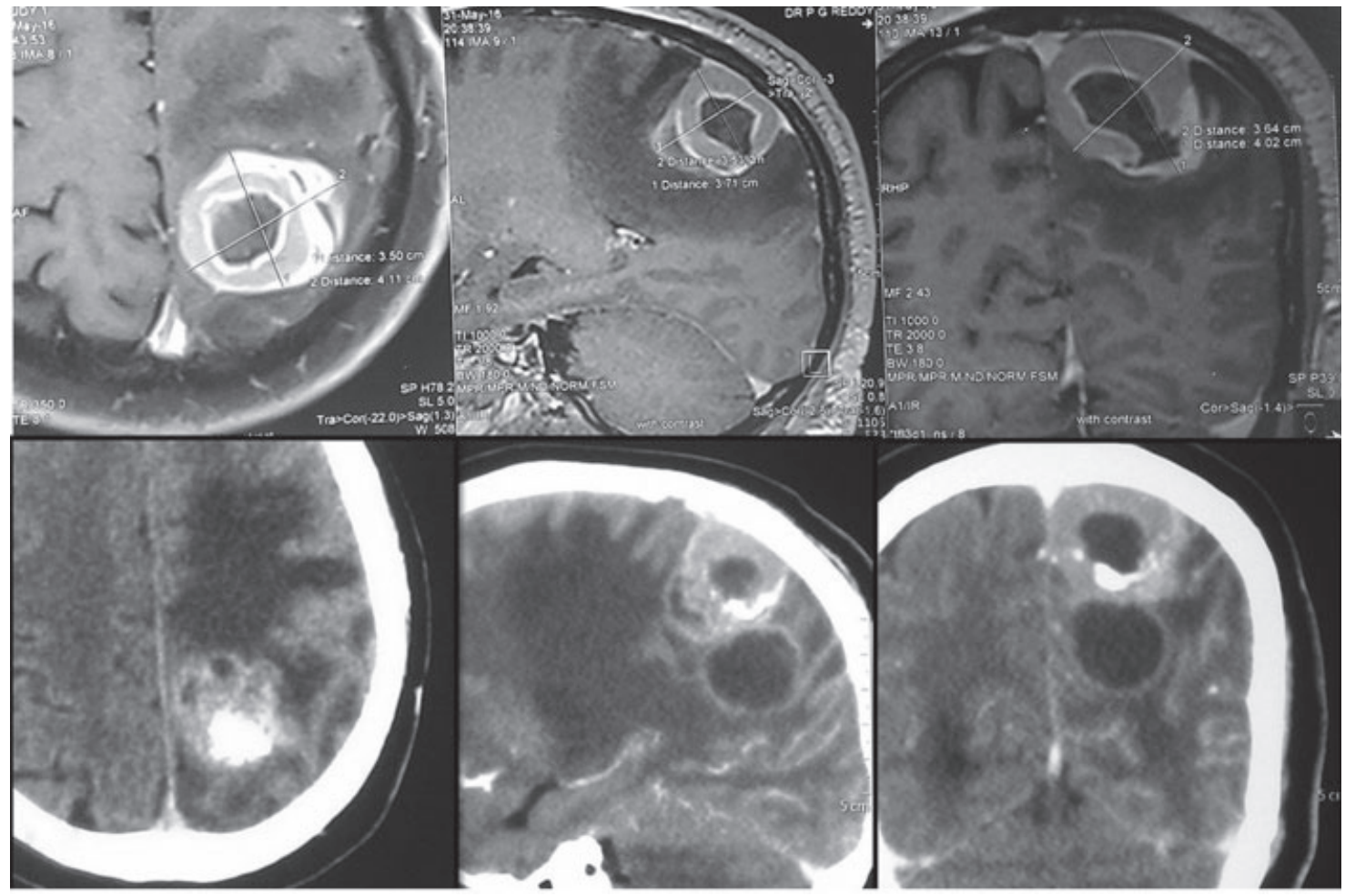

Fig. 1 MRI T1 contrast (first row) and CT of the brain with contrast (second row) done 7 months apart. There is a change in the size, shape, perilesional edema, and pattern of contrast enhancement. The original lesion is thick walled with areas of calcification and the newer lesions are thin walled but incontiguous with the first one.
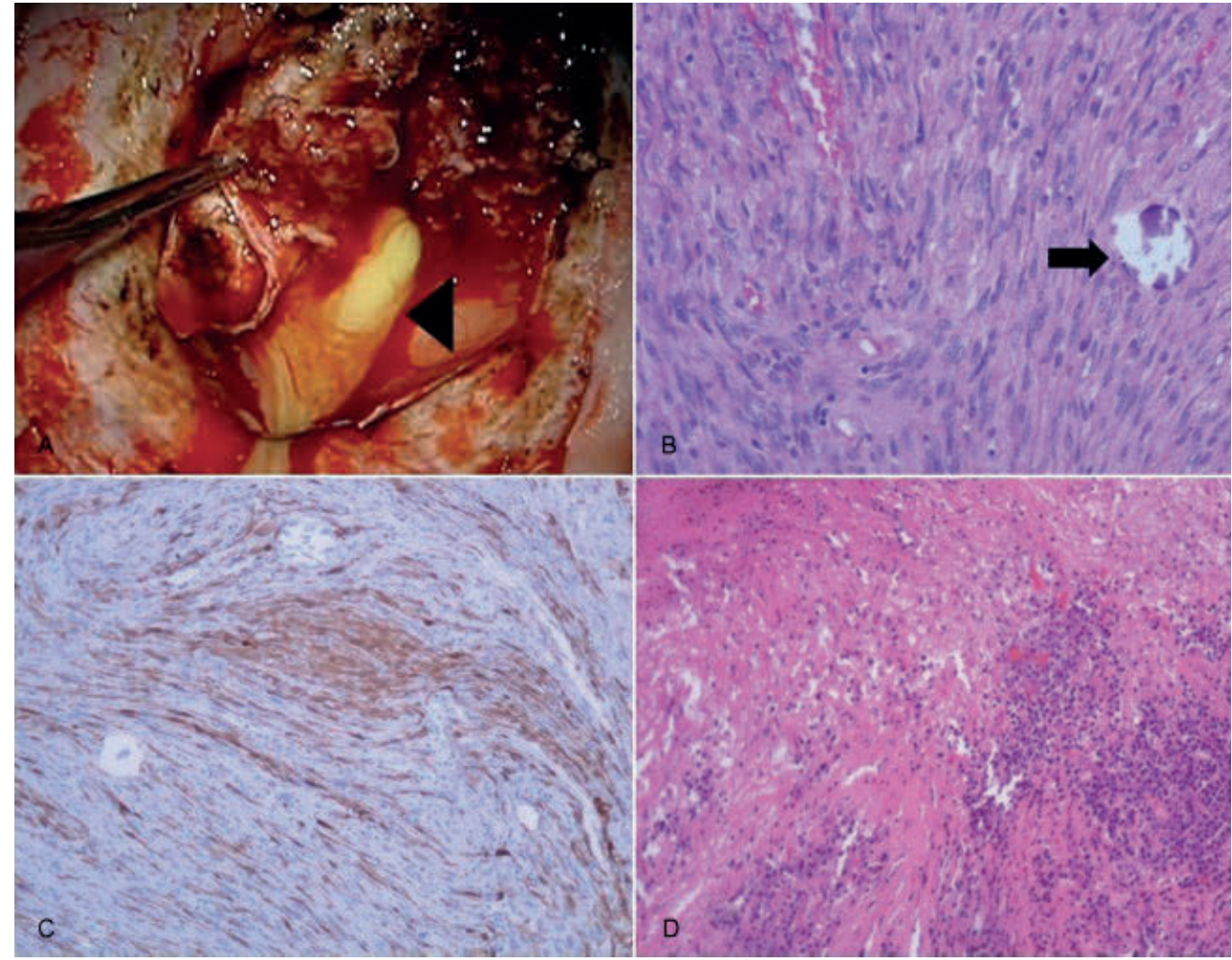

Fig. 2 (A) Intraoperative photograph showing abscess within the tumor (arrow head). (B) H\&E stain showing neoplastic tissue composed of fascicles of cells with oval to spindled nuclei and psammoma body (arrow). (C) Neoplastic cells are positive for epithelial membrane antigen on immunohistochemistry. (D) Dense infiltration by lymphocytes, plasma cells, and histiocytes with focal necrosis also noted in the wall of the tumor. H\&E stain, hematoxylin and eosin stain. 
1H-magnetic resonance spectroscopy (MRS), diffusion-weighted imaging (DWI), dynamic susceptibility contrast perfusion magnetic resonance imaging (MRI), and 99mTc-labeled sulesomab (LeukoScan, Immunomedics, Inc.) are investigations that can aid in differentiating brain abscesses from cystic or necrotic tumors. Lactate (1.3 ppm), acetate $(1.92 \mathrm{ppm})$, and succinate $(2.4 \mathrm{ppm})$ peaks noted on MRS in patients with brain abscess presumably originate from enhanced glycolysis and fermentation of microorganisms. Valine, isoleucine, and leucine $(0.9 \mathrm{ppm})$ peaks in spectroscopic imaging of abscess result from end products of proteolysis by enzymes released by neutrophils in pus. ${ }^{10}$ These peaks also disappear following effective antibiotic therapy and hence, can be used to monitor response to therapy. The spectral metabolite pattern of lactate, amino acids, alanine, and acetate, with or without succinate, shows an abscess caused by obligate anaerobes or a mixture of obligate and facultative anaerobes while that of lactate and amino acids reveals an abscess caused by obligate aerobes or facultative anaerobes. Presence of lactate alone shows small and/or treated abscesses. ${ }^{11}$ Diffusion and apparent diffusion coefficient (ADC) maps are more accurate than MRS in differentiating abscesses from cystic or necrotic tumors. Pyogenic abscesses have high signal on DWI with ADC ratios between 0.45 and 0.8 . Cystic and necrotic portion of tumors have low signal on DWI and ADC values between 15 and $18 .^{10,12}$ Measurement of regional cerebral blood volume (rCBV) by dynamic susceptibility contrast (T2 weighted) MRI hemodynamic imaging reveals high rCBV for high-grade tumors and low rCBV for abscesses compared with normal white matter tracts. ${ }^{13}$ Sulesomab is a monoclonal antibody that binds to antigen present on the surface of activated neutrophils present in the abscess cavity. Absence of tracer uptake in brain lesion on 99mTc-labeled sulesomab (LeukoScan) rules out infection with a high degree of certainty. However, false-negative result may be obtained if the patient is on steroid therapy. ${ }^{14}$

Meningioma with abscess should be considered in the differential diagnosis of dural-based lesions with significant perilesional edema, especially in those with a history of systemic infection. Preoperative evaluation in such patients should include echocardiography, ultrasound of the abdomen and pelvis, dental screening, and, in symptomatic patients, urine culture and ENT (ear-nose-throat) consultation for sinusitis. Radical surgery followed by a suitable antibiotic protocol, deferring adjuvant postoperative therapy till resolution of abscess, is associated with a favorable outcome. ${ }^{14}$

\section{Note}

A written informed consent was obtained from the patient for publication of this case report and any accompanying images.

\section{Funding \\ None.}

\section{Conflict of Interests}

None.

\section{References}

1 Jaiswal AK, Mahapatra AK, Sharma MC. Pituitary abscess associated with prolactinoma. J Clin Neurosci 2004;11(05):533-534

2 Hatiboglu MA, Iplikcioglu AC, Ozcan D. Abscess formation within invasive pituitary adenoma. J Clin Neurosci 2006;13(07):774-777

3 MolièreS, KrémerS. When meningioma becomes anemergency: nocardial brain abscesses superimposed on meningioma. J Neuroradiol 2015;42(04):249-251

4 Lo WB, Cahill J, Carey M, Mehta H, Shad A. Infected intracranial meningiomas. World Neurosurg 2014;81(3-4): 651.e9-651.e13

5 Krishnan SS, Panigrahi M, Pattanagare SG, Varma RD, Rao SI. Abscess within a meningioma: pathogenesis and rare case report. Neurol India 2014;62(02):196-198

6 Young JP, Young PH. Meningioma associated with abscess formation-a case report. Surg Neurol 2005;63 (06):584-585

7 Nassar SI, Haddad FS, Hanbali FS, Kanaan NV. Abscess superimposed on brain tumor: two case reports and review of the literature. Surg Neurol 1997;47 (05):484-488

8 Eisenberg MB, Lopez R, Stanek AE. Abscess formation within a parasagittal meningioma. Case report. J Neurosurg 1998;88 (05):895-897

9 Lind CRP, Muthiah K, Bok APL. Peritumoral Citrobacter koseri abscess associated with parasagittal meningioma. Neurosurgery 2005;57 (04):E814

10 Lai PH, Ho JT, Chen WL, et al. Brain abscess and necrotic brain tumor: discrimination with proton MR spectroscopy and diffusion-weighted imaging. Am J Neuroradiol 2002; 23 (08):1369-1377

11 Lai PH, Li KT, Hsu SS, , et al . Pyogenic brain abscess: findings from in vivo 1.5-T and 11.7-T in vitro proton MR spectroscopy. Am J Neuroradiol 2005;26 (02):279-288

12 Tung GA, Evangelista P, Rogg JM, Duncan JA 3rd. Diffusion-weighted MR imaging of rim-enhancing brain masses. Am J Roentgenol 2001;177:709-712

13 Holmes TM, Petrella JR, Provenzale JM. Distinction between cerebral abscesses and high-grade neoplasms by dynamic susceptibility contrast perfusion MRI. Am J Roentgenol 2004; 183 (05):1247-1252

14 Campennì $\mathrm{A}$, Caruso G, Barresi V, et al. Gliomas with intratumoral abscess formation: description of new cases, review of the literature, and the role of (99m)TC-LeukoScan. Kaohsiung J Med Sci 2015;31 (07):377-383 